\title{
Down-regulation of Notch signaling by a $\gamma$-secretase inhibitor enhances the radiosensitivity of nasopharyngeal carcinoma cells
}

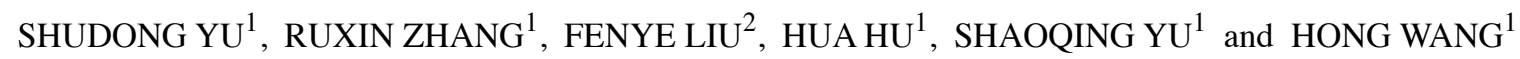 \\ ${ }^{1}$ Department of Otolaryngology, Huadong Hospital, Fudan University, Shanghai 200040; ${ }^{2}$ Department of Traditional \\ Chinese Medicine, Shandong Provincial Hospital Affiliated to Shandong University, Jinan, Shandong 250021, P.R. China
}

Received June 8, 2011; Accepted July 4, 2011

DOI: $10.3892 / o r .2011 .1402$

\begin{abstract}
Currently, the main approach to nasopharyngeal carcinoma (NPC) treatment is radiotherapy (RT), but for many NPC patients, RT is not effective. Increasing RT sensitivity of NPC cells would provide a significant treatment advance for NPC patients. While $\gamma$-secretase inhibitors (GSIs) have gained recent attention as novel anticancer drugs, the mechanism of action of GSIs as radiosensitizers is not well understood. In the present study, radiation-induced anti-proliferative effects of the one GSI (N-[(3,5-difluorophenyl)acetyl]-L-alanyl-2phenyl]glycine-1,1-dimethylethyl ester, DAPT), on CNE2 cells were investigated with the MTT assay; in vitro radiosensitization effects were evaluated by the apoptosis assay and the cell colony formation assay. The activation status of the Notch signaling pathway in DAPT- or dimethyl sulfoxide-treated CNE2 cells was also examined. Notch signaling in NPC cells was found to be down-regulated by DAPT; therefore, DAPT could significantly inhibit CNE2 growth and improve NPC radiosensitization, thus, enhancing $\mathrm{RT}$-induced anti-proliferative effects and apoptosis. Taken together, our data show that Notch signaling down-regulation by GSIs could enhance radiosensitivity of NPC cells, suggesting clinical applications for GSIs as radiosensitizers for NPC therapy.
\end{abstract}

\section{Introduction}

Nasopharyngeal carcinoma (NPC) is a disease with a remarkable geographic and ethnic distribution worldwide, having a high occurrence rate in China and Southeast Asia, especially in the south of China, while being rare in other areas of the world. The observed incidence rates in China and Southeast Asia range from 15 to 50 per 100,000 persons (1). Nasopharyngeal carcinoma arises from epithelial cells that cover the surface and line of the nasopharynx (2). Patients frequently present

Correspondence to: Dr Ruxin Zhang, Department of Otolaryngology, Huadong Hospital, Fudan University, 221 Yan An Xi Road, Shanghai 200040, P.R. China

E-mail: zhangruxinent@126.com

Key words: nasopharyngeal carcinoma, Notch signaling, $\gamma$-secretase inhibitor, radiosensitization with neck masses, nasal symptoms such as epistaxis and nasal obstruction, and otological symptoms such as deafness and tinnitus. Unfortunately, as early-stage symptoms are nonspecific and pathogenic sites can be difficult to examine, most NPC patients are only diagnosed when the tumor has reached an advanced stage.

The main current approach to NPC is radiotherapy (RT). Radiotherapy for NPC is challenging because the nasopharynx is surrounded by an array of radiosensitive anatomical structures, including the brain stem, spinal cord, pituitaryhypothalamic axis, temporal lobes, eyes, middle and inner ears, and parotid glands. Moreover, many NPCs are resistant to RT, resulting in poor prognoses. Therefore, increasing the sensitivity of NPC cells to RT would provide a significant advancement in the effective treatment for NPC patients.

The Notch signaling pathway was first recognized as an important developmental pathway in Drosophila in the first half of the 20th century (3). Many decades later, this pathway has been found to play central roles in stem cell maintenance, cell fate decisions, and regulation of pattern formation. Its dysfunction results in a tremendous variety of developmental defects and adult pathologies (4), such as tumorigenesis. Notably, the Notch signaling pathway is frequently activated in several human malignancies, such as glioma (5), breast cancer (6) and colon cancer (7). It has also been found that the Notch signaling pathway is highly expressed in NPC (8). Further studies have found that Notch signaling was mainly activated in cells expressing embryonic stem cell proteins in human primary NPC (9). On the other hand, the Epstein-Barr virus (EBV), a common contributor to NPC oncogenesis (10), codes for the latent membrane protein 2A (LMP2A), which constitutively activates the Notch pathway in B cells and epithelial cells (11-13). Therefore, the Notch signaling pathway plays important roles in NPC growth promotion and oncogenesis.

$\gamma$-secretase is crucial to the proteolytic activity that releases the Notch intracellular domain; it is thus a central player in the canonical Notch signaling transduction pathway. Therefore, $\gamma$-secretase inhibitors (GSIs) can inhibit Notch signaling. Recently, GSIs have gained attention as novel anti-cancer drugs because of their ability to block the Notch signaling pathway. However, their toxicity toward normal cells and tissues is a major concern; combining GSIs and typical therapies may achieve better curative effects, while reducing side effects (14). In the present study, the effect of the one 
Table I. Examined genes and their PCR primers.

Gene

Primer sequence $5^{\prime} \rightarrow 3$
Amplification size (bp)

Notch1

F: 5'-GCG AGG TCA ACA CAG ACG AG-3'

173

R: 5'-CAG GCA CTT GGC ACC ATT C-3'

Hes-1

F: 5'-TGG AGA GGC GGC TAA GGT GT-3'

124

R: 5'-GCT GGT GTA GAC GGG GAT GAC-3'

$\beta$-actin

F: 5'-CAT GTA CGT TGC TAT CCA GGC-3'

R: 5'-CTC CTT AAT GTC ACG CAC GAT-3'

GSI (N-[(3,5-difluorophenyl)acetyl]-L-alanyl-2-phenyl] glycine-1,1-dimethylethyl ester, DAPT), combined with radiation was investigated; DAPT has been widely used to evaluate the Notch signaling pathway in various cells (15). We found that DAPT could enhance radiosensitivity of NPC cell lines (CNE2) at rather low concentrations.

\section{Materials and methods}

Cell culture and treatment. Human NPC cells (CNE2) were obtained from the Xiangya Central Experiment Laboratory, Central South University, and maintained in RPMI-1640 culture (Hyclone, Logan, USA) supplemented with $10 \%$ fetal bovine serum (FBS, Sijiqing, Hangzhou, China), 100 units/ml penicillin G, and 100 units/ml streptomycin (Gibco, Grand Island, NY). DAPT (Sigma, St. Louis, MO) was dissolved in dimethyl sulfoxide (DMSO, Amresco, Solon, $\mathrm{OH}$ ) to yield a final concentration equal to $5 \mathrm{mmlol} / \mathrm{l}$. Cells were then treated with various concentrations of DAPT or DMSO of the same volume (control).

Radiation. Each sample received a single dose of 0, 1, 2, 3, 4, 6 or $8 \mathrm{~Gy}$ at $400 \mathrm{cGy} / \mathrm{min}$, using a linear accelerator (Trilogy, Austin, TX, USA) with $6 \mathrm{MV}$-photons at room temperature.

MTT assay. First, the effects of DAPT on cell proliferation were determined by the MTT assay. CNE2 cells (1000 per well) were plated in 96-well plates, allowed to adhere overnight, and treated with various concentrations of DAPT or DMSO for $1-3$ days at $37^{\circ} \mathrm{C}$; MTT assays were then carried out. In vitro growth inhibitory effects of DAPT + RT on CNE2 cells was also assessed using MTT assays as previously described (16). Briefly, cells in 96-well plates were treated with $10 \mu \mathrm{mol} / 1$ DAPT for $48 \mathrm{~h}$, and then radiated with 4 Gy as described above. Every $24 \mathrm{~h}$ for 4 days, MTT assays were carried out; MTT dye was added to each well and incubated for $2 \mathrm{~h}$ at $37^{\circ} \mathrm{C}$ according to the manufacturer's guidelines (GenMed, Shanghai, China). Cell suspension absorbance was measured at $570 \mathrm{~nm}$ using a microplate reader (Bio-Tek Inc., USA); the background absorbance of the cell-free medium was subtracted.

Apoptosis assay. The percentage of apoptotic cells was assessed using flow cytometry (FCM) with the Annexin V-FITC/propidium iodide (PI) method. Cultured CNE2 cells were randomly assigned to four groups: DMSO, DAPT, DAPT + radiotherapy and radiotherapy groups. Before the apoptosis assay, CNE2 cells were incubated in $35-\mathrm{mm}$ dishes with $10 \mu \mathrm{mol} / 1 \mathrm{DAPT}$ or DMSO of the same volume for 2 days; apoptotic cell percentages in the DMSO and DAPT groups were then assessed. The DAPT + radiotherapy and radiotherapy groups cells were irradiated (2 Gy) with a linear accelerator. After radiation, medium was exchanged for normal medium and cultured for 2 days. Apoptotic cell percentages in DAPT + radiotherapy and radiotherapy groups were then assessed, following the manufacturer's instructions (Biouniquer, China). Briefly, unfixed cultured cells were washed twice with cold PBS; $1-5 \times 10^{5}$ cells were collected, resuspended in binding buffer and incubated with Annexin V-FITC in buffer containing PI for $10 \mathrm{~min}$ at room temperature in the dark. Cells were then analyzed within $1 \mathrm{~h}$, using FCM (Epics Altra; Beckman Coulter, Fullerton, CA).

Cell colony formation assay. Before the cell colony formation assay, cultured CNE2 cells were randomly assigned to one of two groups: radiotherapy alone or DAPT + radiotherapy. Cells were trypsinized to generate a single-cell suspension; a specified number of cells were seeded into each well of 6-well tissue culture plates according to their respective radiation doses, and allowed to attach overnight. The DAPT + radiotherapy cells were then incubated with $10 \mu \mathrm{mol} / 1 \mathrm{DAPT}$ for $48 \mathrm{~h}$, while radiotherapy cells were incubated with the same volume of DMSO for $48 \mathrm{~h}$. All cells were irradiated $(0,1,2,3,4,6$ or $8 \mathrm{~Gy})$ in the presence of DAPT or DMSO, using a linear accelerator. After radiation, the medium was changed to normal medium; cells were allowed to grow for 2 weeks. Methanol was used to fix the cells, which were dyed with Giemsa. After air drying, the visible colonies were counted. The surviving fraction was calculated as: [(mean colonies counted)/(cells plated) $\mathrm{x}$ (plating efficiency)]; plating efficiency was defined as: [(mean colonies counted)/(cells plated)] for unradiated controls ( $0 \mathrm{~Gy})$.

Real-time PCR. Total RNA was extracted from specimens using the TRIzol reagent (Invitrogen, Carlsbad, CA) and converted to cDNA using reverse transcriptase MMLV (Promega, Madison, WI). Aliquots of cDNA were subjected to quantitative real-time PCR using a StepOne plus Real-time PCR system (Applied Biosystems, Foster City, CA). Primer sequences used are listed in Table I; mRNA levels were normalized relative to $\beta$-actin mRNA levels.

Western blot analysis. Western blot analyses were performed as previously described (17). Briefly, concentrations of protein 

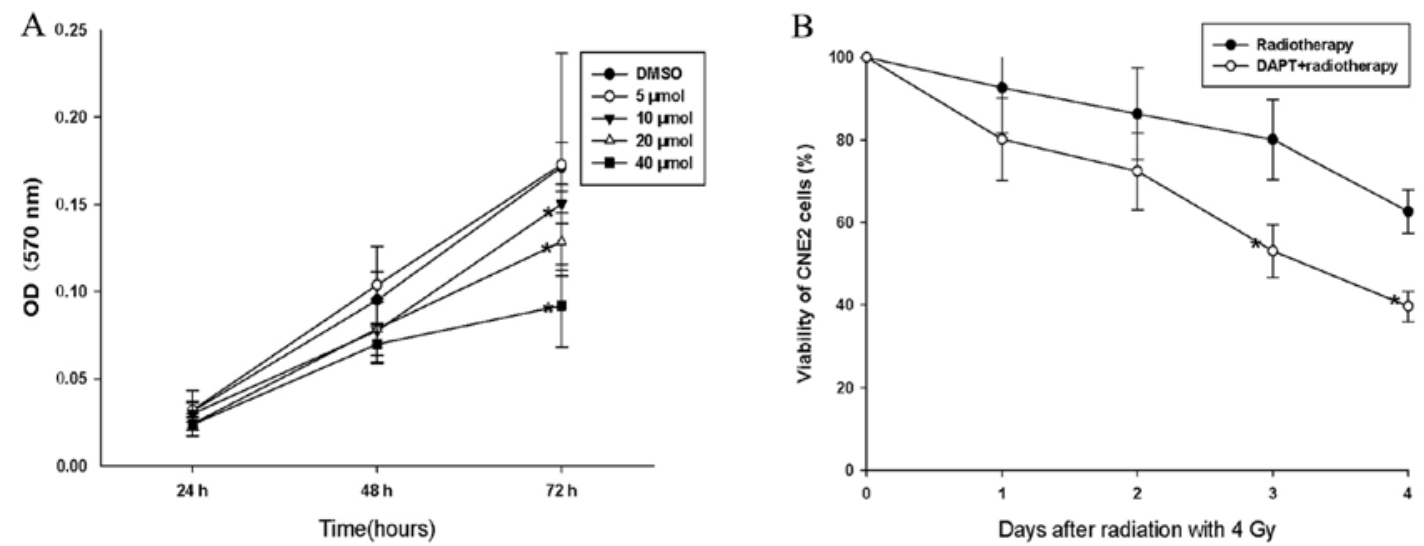

Figure 1. DAPT inhibits CNE2 growth of CNE2 and enhances the anti-proliferation ability of radiation. Cells were pretreated with DAPT at the indicated concentrations for 24-72 h; proliferation was measured by the MTT assay. ${ }^{*} \mathrm{P}<0.05$ compared to DMSO groups. CNE2 cells were pretreated with $10 \mu$ mol/1 DAPT or DMSO for $48 \mathrm{~h}$ and irradiated at $4 \mathrm{~Gy}$; viability was measured by the MTT assay (B). ${ }^{*} \mathrm{P}<0.05$ compared to radiotherapy groups.
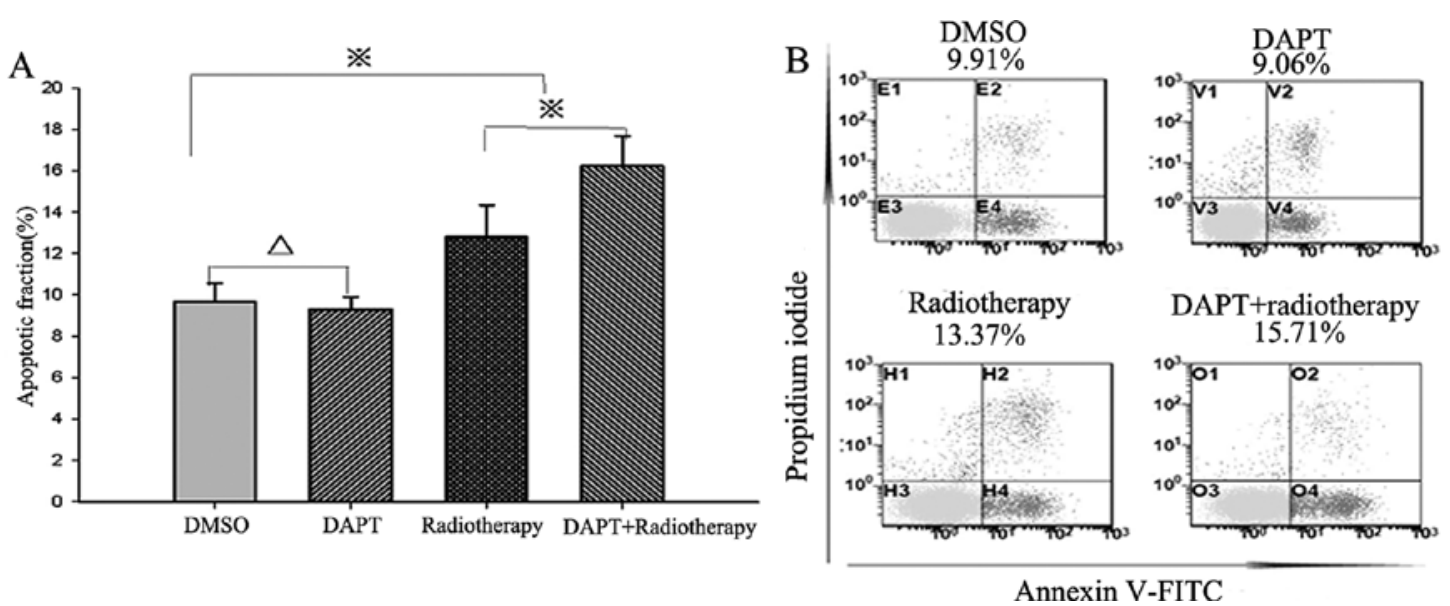

Figure 2. DAPT enhanced the radiation-induced apoptotic fraction in CNE2 cells. (A) Apoptosis fraction induced by DAPT + radiotherapy was significantly higher than that induced by irradiation alone ( $\left.{ }^{*} \mathrm{P}<0.05\right)$; no significant difference was found between the DAPT and the DMSO groups $\left({ }^{\wedge} \mathrm{P}>0.05\right)$. Apoptotic fractions of the radiotherapy groups (radiotherapy and DAPT + radiotherapy) differed from those of the DMSO groups $(\mathrm{P}<0.05)$. (B) Representative FCM examples.

extracted from DAPT- or DMSO-pretreated CNE2 cells were determined by the Lowry method. Equal amounts of protein were separated by $10 \%$ SDS-PAGE electrophoresis and electrophoretically transferred to PVDF membranes (Millipore, Bedford, MA). Rabbit anti-human Notch1 intracellular domain (NICD) antibody (1:500; Cell Signaling Technology, Boston, MA) and mouse antihuman Hes-1 antibody (1:500; Santa Cruz Biotechnology, Santa Cruz, CA) were used to detect the expression of cleaved Notch1 and Hes-1. $\beta$-actin and Erk were used as an internal controls.

Statistical analysis. Each test was repeated three times. In the cell colony formation assay, a multi-target single-hit mathematical model was used to draw a survival curve with SigmaPlot 11.0 (Systat Software Inc., San Jose, CA); cellular radiosensitivity $\left(D_{0}\right)$, capacity for sublethal damage repair $\left(D_{q}\right)$, survival fraction at a dose of $2 \mathrm{~Gy}\left(\mathrm{SF}_{2}\right)$, and the extrapolation number $(\mathrm{N})$ were obtained, and each group's radiosensitivity was analyzed and compared. Other data were analyzed for statistical significance using analysis of variance (ANOVA) or Student's t-test with SPSS 17.0 software (SPSS, Chicago, IL). $\mathrm{P}<0.05$ was considered statistically significant.

\section{Results}

DAPT inhibits CNE2 growth and enhances the anti-proliferation effect of radiation. To determine whether the $\gamma$-secretase inhibitor (DAPT) can inhibit NPC cell growth, and to select the lowest effective concentration, we examined its effect on CNE2 cell proliferation. Cells were treated with various concentrations of DAPT $(0,5,10,20$ or $40 \mu \mathrm{mol} / \mathrm{l})$. Results revealed that DAPT dose-dependently inhibited CNE2 cell growth (Fig. 1A); $10 \mu \mathrm{mol} / 1$ was the lowest concentration to inhibit proliferation after incubation for $72 \mathrm{~h}(\mathrm{P}<0.05)$, but no DAPT concentrations affected CNE2 cell proliferation at 24 or $48 \mathrm{~h}(\mathrm{P}>0.05)$. To study the inhibitory effects of DAPT + radiation on CNE2 cells, we pretreated cells with $10 \mu \mathrm{mol} / 1$ DAPT or DMSO for $48 \mathrm{~h}$. After radiation at $4 \mathrm{~Gy}$ for 3 days, CNE2 cell viability in the DAPT + radiotherapy group differed from that in radiotherapy group $(\mathrm{P}<0.05$, Fig. 1B). These results show that DAPT can inhibit CNE2 growth and enhance the anti-proliferative effects of radiation.

DAPT enhances RT-induced apoptosis. Because DAPT inhibits CNE2 growth and enhances the anti-proliferation 

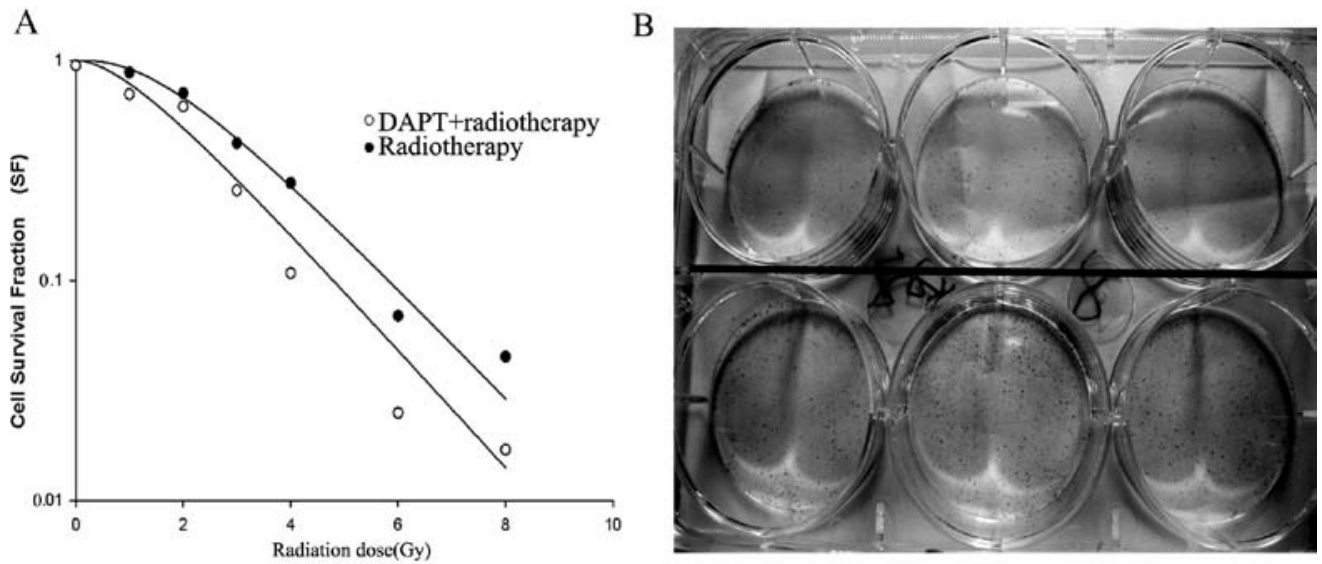

Figure 3. Clonogenic assay to obtain the single-dose radiation survival curves of CNE2. (A) Radiation survival curves for CNE2 cells pretreated with $10 \mu$ mol/1 of DAPT or DMSO for 2 days. Data points represent mean of 3 experiments. (B) CNE2 cells radiated at 8 Gy showed visible colonies after 2 weeks. The 3 upper wells were DAPT + radiotherapy groups; the 3 lower wells were radiotherapy groups; DAPT + radiotherapy groups had fewer visible colonies than did radiotherapy groups.

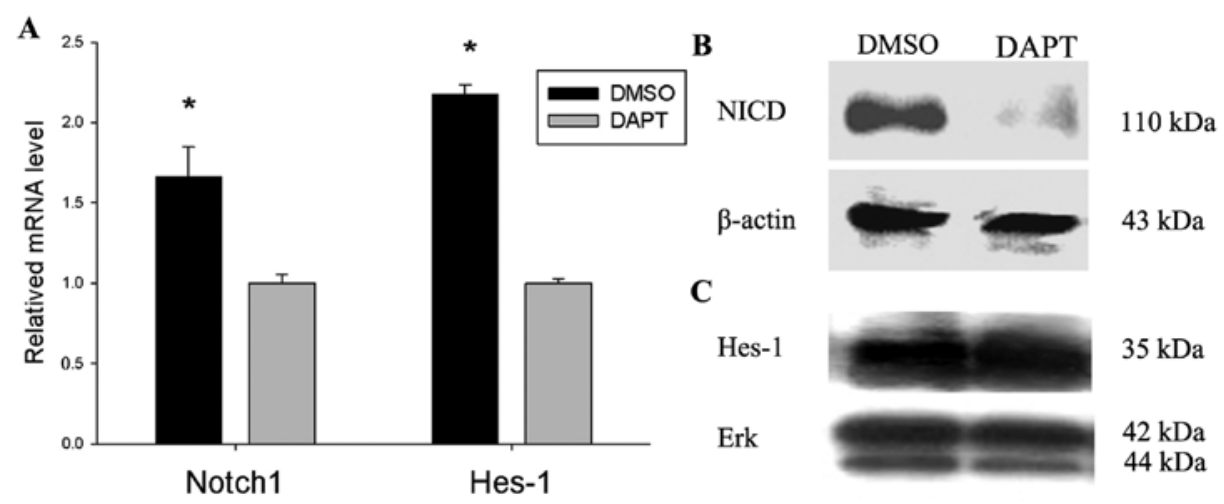

Figure 4. Expression of Notch1 and Hes-1 in DAPT- or DMSO-treated CNE2 cells (A) Notch1 and Hes-1 mRNA levels was detected by real-time PCR in DAPT- or DMSO-treated cells. Error bars represent the standard deviation of 3 independent experiments ("P<0.01). (B) Western blot analysis demonstrates that DAPT could decrease NICD expression. $\beta$-actin expression shows equivalent protein loading. (C) Western blot analysis demonstrates that DAPT could decrease Hes-1 expression. Erk expression shows equal protein loading.

capacity of radiation (as shown above), we considered that it may increase RT-induced apoptosis; therefore, we investigated RT-induced apoptosis in DMSO- and DAPT-treated CNE2 cells. As shown in Fig. 2, significantly greater RT-induced apoptosis was observed in the DAPT-treated cells (DAPT + radiotherapy group) than in the DMSO-treated cells (radiotherapy group; $\mathrm{P}<0.05$ ). Two days after 2 Gy radiotherapy, the apoptotic fraction of the radiotherapy group cells was $\sim 12.8 \%$, but that of cells pretreated with DAPT for 2 days (DAPT + radiotherapy group) was $\sim 16.2 \%$. The apoptotic fraction of the two groups (radiotherapy and DAPT + radiotherapy groups) was different from that of the DMSO groups $(\mathrm{P}<0.05)$. To ascertain whether apoptosis was induced by DAPT, we also measured apoptosis in cells that were pretreated with DMSO or DAPT for 2 days. As shown in Fig. 2, DAPT had no obvious impact on the apoptotic fraction when the cells were only treated for 2 days ( $\mathrm{P}>0.05$ ), showing that Notch inhibition using DAPT sensitizes NPC cells to radiation-induced apoptosis.

DAPT improves radiosensitization of NPC cells. To further confirm the radiosensitizing effect of DAPT at other radiation doses, we measured the clonogenic survival of CNE2 cells. A multitarget single-hit model was used to calculate cellular radiosensitivity $\left(D_{0}\right)$, capacity for sublethal damage repair $\left(D_{q}\right)$, survival fraction at a dose of $2 \mathrm{~Gy}\left(\mathrm{SF}_{2}\right)$, and the extrapolation number $(\mathrm{N})(18,19)$. The survival curve parameters are listed in Table II. In this model, SF is calculated as follows: SF = $1-\left(1-\mathrm{e}^{-\mathrm{KD}}\right)^{\mathrm{N}}$. All radiotherapy group parameters were greater than those of the DAPT + radiotherapy groups, indicating that DAPT makes CNE2 cells more sensitive to radiation (Fig. 3A). Results could be easily seen with the naked eye (Fig. 3B); there were few visible colonies in cells that were pretreated with DAPT and radiated with $8 \mathrm{~Gy}$.

DAPT inhibits Notch signaling in CNE2 cells. To further determine the underlying mechanism of DAPT's enhancement of NPC radiosensitivity, the activation status of Notch signaling in DAPT- or DMSO-treated CNE2 cells was examined with real-time PCR and Western blotting. In the Notch signaling pathway, NICD is the activated form of Notch1 and is cleaved by $\gamma$-secretase from the membrane; Hes- 1 is the downstream target gene of Notch signaling. As expected, Notch1 and Hes-1 
Table II. Survival curve parameters fitting the data into a multitarget single-hit model.

\begin{tabular}{lcccc}
\hline Cell & $\mathrm{D}_{0}$ & $\mathrm{D}_{\mathrm{q}}$ & $\mathrm{SF}_{2}$ & $\mathrm{~N}$ \\
\hline Radiotherapy & 1.7292 & 3.441 & 0.6768 & 2.9902 \\
DAPT + radiotherapy & 1.6197 & 1.5795 & 0.4929 & 1.9752
\end{tabular}

$\mathrm{D}_{0}$, cellular radiosensitivity; $\mathrm{D}_{\mathrm{q}}$, capacity for sublethal damage repair; $\mathrm{SF}_{2}$, survival fraction at a dose of $2 \mathrm{~Gy} ; \mathrm{N}$, the extrapolation number.

expression, both mRNA and protein, were highly activated in DMSO-treated cells compared to DAPT-treated cells (Fig. 4). These results suggest that DAPT significantly down-regulates Notch1 and Hes-1 expression.

\section{Discussion}

Radiotherapy is the current mainstay of NPC treatment. Enhancing the radiosensitivity of NPC cells is of great importance in overcoming this disease. Here, we show that GSIs can significantly down-regulate Notch signaling and enhance radiosensitivity in NPC cells.

The widely conserved Notch signaling pathway influences key aspects of normal development including differentiation, proliferation and apoptosis (20). Mammals, including humans, possess 4 Notch proteins (Notch 1-4), and 5 Notch ligands (Delta-like 1, 3 and 4 and Jagged 1 and 2) $(21,22)$. Signaling occurs when the ligands bind and interact with Notch, inducing a series of cleavages, $\mathrm{S} 2, \mathrm{~S} 3$, and $\mathrm{S} 4$. Of these, the S3/4 cleavage is intramembranous and mediated by a presenilin-dependent $\gamma$-secretase, causing translocation of NICD into the nucleus, where it then interacts with a transcriptional factor CSL (CBF1/RBPJ $\kappa$ in mammals), activating downstream target genes, such as Hes-1 $(23,24)$.

Targeting Notch signaling with GSIs or siRNA is a novel way to control tumors with hyperactive Notch signaling. The advantage of using GSIs lies in the fact that all Notch receptors require $\gamma$-secretase for processing and signaling (25). On the other hand, GSIs can be easily used clinically. In this study, we used DAPT to inhibit Notch signaling. Although the effects of GSIs on various tumors are widely reported (26-28), knowledge of their roles as radiosensitizers is limited. Here, we found that GSIs can enhance the radiosensitivity of NPC cells.

The concept of radiosensitizers was first proposed in 1958 (29). They include traditional chemotherapeutic agents and some newer molecular targeting agents $(30,31)$, which are widely used and considered to improve local-regional radiotherapy effects (32). These chemotherapeutic agents include 5-fluorouracil, platinum analogs, gemcitabine and DNA topoisomerase I-targeting drugs. While older agents focus on DNA as their target, some newer agents that do not target DNA can also act as radiosensitizers; they include the epidermal growth factor receptor blockers, farnesyltransferase inhibitors, and COX-2 inhibitors (31). As novel anti-cancer drugs, Notch inhibitors should be used as radiosensitizers in treating NPC.

Why inhibit Notch signaling by GSIs to enhance NPC radiation sensitivity? As mentioned above, the Notch signaling pathway plays critical roles in NPC growth and oncogenesis. When Notch signaling is inhibited, NPC cells become less viable and more sensitive to radiation. Moreover, Notch is a critical pathway in cancer stem cells (CSCs); inhibition of the Notch signaling pathway could deplete CSCs and inhibit tumor growth (33-37). Moreover, CSCs contribute to radiation resistance (38-40); NPC CSCs are confirmed to be resistant to radiation. Previous studies have found that drugs or methods that target CSCs can overcome radiation resistance (41-43). When Notch signaling is inhibited, the proportion of CSCs may decrease, which can also make NPC cells more radiosensitive. In further investigations, we will explore the effects of GSIs on CSCs proportion.

In conclusion, our data indicate that the down-regulation of Notch signaling by a $\gamma$-secretase inhibitor can enhance the radiosensitivity of NPC cells. Notch inhibitors may have promising clinical applications as radiosensitizers in the treatment of NPC.

\section{Acknowledgements}

This study was supported by the Scientific Research Foundation of the Shanghai Health Bureau (no. 2007002). The authors thank Weiqiang Ge and Guoping Zhang for technical support in radiotherapy and flow cytometry. We also thank Yanqing Wang for her laboratory assistance.

\section{References}

1. Cao JX, Cui YX, Long ZJ, et al: Pluripotency-associated genes in human nasopharyngeal carcinoma CNE-2 cells are reactivated by a unique epigenetic sub-microenvironment. BMC Cancer 10: $68,2010$.

2. Brennan B: Nasopharyngeal carcinoma. Orphanet J Rare Dis 1: 23, 2006.

3. Purow B: Notch inhibitors as a new tool in the war on cancer: a pathway to watch. Curr Pharm Biotechnol 10: 154-160, 2009.

4. Lai EC: Notch signaling: control of cell communication and cell fate. Development 131: 965-973, 2004.

5. Jiang L, Wu J, Chen Q, Hu X, Li W and Hu G: Notch1 expression is upregulated in glioma and is associated with tumor progression. J Clin Neurosci 18: 387-390, 2011.

6. Guo S, Liu M and Gonzalez-Perez RR: Role of Notch and its oncogenic signaling crosstalk in breast cancer. Biochim Biophys Acta 1815: 197-213, 2011.

7. Zhang Y, Li B, Ji ZZ and Zheng PS: Notch1 regulates the growth of human colon cancers. Cancer 116: 5207-5218, 2010.

8. Wang M, Li JT, Zeng YX, Hou JH and Lin QQ: Expression and significance of Notch1, P21WAF1 and involucrin in nasopharyngeal carcinoma. Ai Zheng 24: 1230-1234, 2005.

9. Zhang Y, Peng J, Zhang $\mathrm{H}$, et al: Notch1 signaling is activated in cells expressing embryonic stem cell proteins in human primary nasopharyngeal carcinoma. J Otolaryngol Head Neck Surg 39: 157-166, 2010.

10. Li DJ, Bei JX, Mai SJ, et al: The dominance of China 1 in the spectrum of Epstein-Barr virus strains from Cantonese patients with nasopharyngeal carcinoma. J Med Virol 81: 1253-1260, 2009.

11. Anderson LJ and Longnecker R: An auto-regulatory loop for EBV LMP2A involves activation of Notch. Virology 371: 257-266, 2008.

12. Anderson LJ and Longnecker R: Epstein-Barr virus latent membrane protein $2 \mathrm{~A}$ exploits Notch1 to alter B-cell identity in vivo. Blood 113: 108-116, 2009.

13. Pang MF,Lin KW and Peh SC: The signaling pathways of EpsteinBarr virus-encoded latent membrane protein 2A (LMP2A) in latency and cancer. Cell Mol Biol Lett 14: 222-247, 2009.

14. Lin J, Zhang XM, Yang JC, Ye YB and Luo SQ: gamma-secretase inhibitor-I enhances radiosensitivity of glioblastoma cell lines by depleting CD133+ tumor cells. Arch Med Res 41: 519-529, 2010. 
15. Jing W, Xiong Z, Cai X, et al: Effects of gamma-secretase inhibition on the proliferation and vitamin $\mathrm{D}(3)$ induced osteogenesis in adipose derived stem cells. Biochem Biophys Res Commun 392: 442-447, 2010.

16. Zellweger T, Chi K, Miyake H, Adomat H, Kiyama S, Skov K and Gleave ME: Enhanced radiation sensitivity in prostate cancer by inhibition of the cell survival protein clusterin. Clin Cancer Res 8: 3276-3284, 2002.

17. Shi GM, Xu Y, Fan J, et al: Identification of side population cells in human hepatocellular carcinoma cell lines with stepwise metastatic potentials. J Cancer Res Clin Oncol 134: 1155-1163, 2008.

18. Krarup M, Poulsen HS and Spang-Thomsen M: Cellular radiosensitivity of small-cell lung cancer cell lines. Int J Radiat Oncol Biol Phys 38: 191-196, 1997.

19. Xu QY, Gao Y, Liu Y, Yang WZ and Xu XY: Identification of differential gene expression profiles of radioresistant lung cancer cell line established by fractionated ionizing radiation in vitro. Chin Med J (Engl) 121: 1830-1837, 2008.

20. Sjolund J, Manetopoulos C, Stockhausen MT and Axelson H: The Notch pathway in cancer: differentiation gone awry. Eur J Cancer 41: 2620-2629, 2005.

21. Diao JS, Zhang X, Xia WS, et al: Aberrant Notch signaling: a potential pathomechanism of vitiligo. Med Hypotheses 73: 70-72, 2009.

22. Dufraine J, Funahashi Y and Kitajewski J: Notch signaling regulates tumor angiogenesis by diverse mechanisms. Oncogene 27: 5132-5137, 2008.

23. Nam Y, Aster JC and Blacklow SC: Notch signaling as a therapeutic target. Curr Opin Chem Biol 6: 501-509, 2002.

24. Tien AC, Rajan A and Bellen HJ: A Notch updated. J Cell Biol 184: 621-629, 2009.

25. Suwanjunee $S$, Wongchana $W$ and Palaga T: Inhibition of gammasecretase affects proliferation of leukemia and hepatoma cell lines through Notch signaling. Anticancer Drugs 19: 477-486, 2008.

26. Curry CL, Reed LL, Golde TE, Miele L, Nickoloff BJ and Foreman KE: Gamma secretase inhibitor blocks Notch activation and induces apoptosis in Kaposi's sarcoma tumor cells. Oncogene 24: 6333-6344, 2005.

27. Hallahan AR, Pritchard JI, Hansen S, et al: The SmoA1 mouse model reveals that notch signaling is critical for the growth and survival of sonic hedgehog-induced medulloblastomas. Cancer Res 64: 7794-7800, 2004

28. O'Neil J, Calvo J, McKenna K, et al: Activating Notch1 mutations in mouse models of T-ALL. Blood 107: 781-785, 2006.

29. Heidelberger C, Griesbach L, Cruz O, Schnitzer RJ and Grunberg E: Fluorinated pyrimidines. VI. Effects of 5-fluorouridine and 5-fluoro-2'-deoxyuridine on transplanted tumors. Proc Soc Exp Biol Med 97: 470-475, 1958.
30. Spalding AC and Lawrence TS: New and emerging radiosensitizers and radioprotectors. Cancer Invest 24: 444-456, 2006.

31. Kvols LK: Radiation sensitizers: a selective review of molecules targeting DNA and non-DNA targets. J Nucl Med 46 (Suppl 1): S187-S190,2005.

32. Cooper JS and Ang KK: Concomitant chemotherapy and radiation therapy certainly improves local control. Int J Radiat Oncol Biol Phys 61: 7-9, 2005.

33. Zhang XP, Zheng G, Zou L, et al: Notch activation promotes cell proliferation and the formation of neural stem cell-like colonies in human glioma cells. Mol Cell Biochem 307: 101-108, 2008.

34. Sikandar SS, Pate KT, Anderson S, Dizon D, Edwards RA, Waterman ML and Lipkin SM: NOTCH signaling is required for formation and self-renewal of tumor-initiating cells and for repression of secretory cell differentiation in colon cancer. Cancer Res 70: 1469-1478, 2010.

35. Fan X, Khaki L, Zhu TS, et al: NOTCH pathway blockade depletes CD133-positive glioblastoma cells and inhibits growth of tumor neurospheres and xenografts. Stem Cells 28: 5-16, 2010.

36. Harrison H, Farnie G, Howell SJ, et al: Regulation of breast cancer stem cell activity by signaling through the Notch4 receptor. Cancer Res 70: 709-718, 2010.

37. Zhen Y, Zhao S, Li Q, Li Y and Kawamoto K: Arsenic trioxidemediated Notch pathway inhibition depletes the cancer stem-like cell population in gliomas. Cancer Lett 292: 64-72, 2010.

38. Bao S, Wu Q, McLendon RE, et al: Glioma stem cells promote radioresistance by preferential activation of the DNA damage response. Nature 444: 756-760, 2006.

39. Phillips TM, McBride WH and Pajonk F: The response of $\mathrm{CD} 24\left(-/\right.$ low $/ \mathrm{CD} 44^{+}$breast cancer-initiating cells to radiation. J Natl Cancer Inst 98: 1777-1785, 2006.

40. Woodward WA, Chen MS, Behbod F, Alfaro MP, Buchholz TA and Rosen JM: WNT/beta-catenin mediates radiation resistance of mouse mammary progenitor cells. Proc Natl Acad Sci USA 104: 618-623, 2007.

41. Teimourian S, Jalal R, Sohrabpour M and Goliaei B: Downregulation of Hsp27 radiosensitizes human prostate cancer cells. Int J Urol 13: 1221-1225, 2006.

42. Kurrey NK, Jalgaonkar SP, Joglekar AV, Ghanate AD, Chaskar PD, Doiphode RY and Bapat SA: Snail and slug mediate radioresistance and chemoresistance by antagonizing p53-mediated apoptosis and acquiring a stem-like phenotype in ovarian cancer cells. Stem Cells 27: 2059-2068, 2009.

43. Zhang X, Komaki R, Wang L, Fang B and Chang JY: Treatment of radioresistant stem-like esophageal cancer cells by an apoptotic gene-armed, telomerase-specific oncolytic adenovirus. Clin Cancer Res 14: 2813-2823, 2008. 